\title{
Generalized hook lengths in symbols and partitions
}

\author{
Christine Bessenrodt • Jean-Baptiste Gramain • \\ Jørn B. Olsson
}

Received: 11 April 2011 / Accepted: 22 November 2011 / Published online: 8 December 2011

(C) Springer Science+Business Media, LLC 2011

\begin{abstract}
In this paper we present, for any integer $d$, a description of the set of hooks in a $d$-symbol. We then introduce generalized hook length functions for a $d$-symbol, and prove a general result about them, involving the core and quotient of the symbol. We list some applications, for example to the well-known hook lengths in integer partitions. This leads in particular to a generalization of a relative hook formula for the degree of characters of the symmetric group discovered by G. Malle and G. Navarro in Trans. Am. Math. Soc. 363, 6647-6669, 2011.
\end{abstract}

Keywords Symbols · Hooks · Hook lengths · Partitions · Core · Quotient

The celebrated hook formula for the degrees of the irreducible characters of the finite symmetric groups has been a source of inspiration for several other degree formulas. In his work [2] on unipotent degrees in reflection groups G. Malle used $d$-symbols as labels, defined hooks in $d$-symbols and associated a length to a hook. With these he was able to prove a "hook formula" for the degrees. He also proved formulas involving suitable cores and quotients of symbols.

C. Bessenrodt

Institut für Algebra, Zahlentheorie und Diskrete Mathematik, Leibniz Universität Hannover,

Welfengarten 1, 30167 Hannover, Germany

e-mail: bessen@math.uni-hannover.de

J.-B. Gramain

Institute of Mathematics, University of Aberdeen, King's College, Fraser Noble Building, Aberdeen AB24 3UE, UK

e-mail: jbgramain@abdn.ac.uk

J.B. Olsson ( $₫)$

Department of Mathematical Sciences, University of Copenhagen, Universitetsparken 5, 2100

Copenhagen $\varnothing$, Denmark

e-mail: olsson@math.ku.dk 
Considering here for simplicity an $h$-hook as a pair $(a, b)$ of integers satisfying $0 \leq b<a$ and $a-b=h$, an $h$-hook may contribute a factor $h$ (for characters of the symmetric groups), or $q^{h}-1$ (for unipotent characters in general linear groups over $G F(q)$ ), or more generally $q^{h}-\zeta$ for some complex root of unity $\zeta$ to the degree of a character. Thus knowledge of the number of $h$-hooks in a partition or a symbol for all $h$ gives information about character degrees.

This paper is concerned with ways of organizing hooks which lead to alternative versions of hook formulas. The hope is that these versions may be better suited to deal with explicit degree problems.

We introduce generalized hook length functions for $d$-symbols and prove a general result about them. More specifically we consider certain functions $h$ from the set $H(S)$ of hooks of a $d$-symbol $S$ to $\mathbb{R}$ and decompositions of the multiset $\mathcal{H}(S)$ of all generalized hook lengths $h(z)$, where $z \in H(S)$. For a given positive integer $\ell$ we find a decomposition which is compatible with the $\ell$-core and $\ell$-quotient of $S$.

Based on a crucial well controlled correspondence between hooks in symbols and hooks in associated core and quotient symbols, our main result (Theorem 3.2) on generalized hook lengths for symbols is deduced. This may seem quite a special and technical result, but its consequence Theorem 3.3 really provides a very general and unified approach to hooks. It has a number of applications. For instance, we show that the relative hook formula obtained by Malle and Navarro [3, Theorem 9.1] is just the well-known hook formula for the degree of the irreducible characters of the symmetric groups with the hooks suitably arranged (Remark 4.12). If $\mathcal{H}(\lambda)$ is the multiset of hook lengths for a partition $\lambda$, and $\lambda$ has $d$-core partition $\lambda_{(d)}$ then we have in particular $\mathcal{H}\left(\lambda_{(d)}\right) \subset \mathcal{H}(\lambda)$. Furthermore the remaining elements of $\mathcal{H}(\lambda)$ may be seen as modified hook lengths of a $d$-quotient partition for $\lambda$ (Theorem 4.7; see Example 4.8 for an illustration).

Our paper is organized as follows. In Sect. 1 we explain the relations between partitions, $\beta$-sets and $d$-symbols, their hooks and their corresponding cores and quotients. In Sect. 2 we set up useful bijections between the set of hooks of a symbol and the set of hooks of its core and quotient. This is used in Sect. 3 to prove our key result, Theorem 3.3. The theorem is then applied to partitions in Sect. 4 and to symbols in Sect. 5. The main results of the final section may be found in a somewhat different form in [2, Sect. 3] (especially the subsection on Harish-Chandra theory).

\section{On $\beta$-sets and $d$-symbols}

A $\beta$-set $X$ is a finite subset of $\mathbb{N}_{0}$. For $s \in \mathbb{N}_{0}$ we put $X^{+s}=(X+s) \cup\{0,1, \ldots$, $s-1\}$. Write $X=\left\{a_{1}, a_{2}, \ldots, a_{t}\right\}$ where we always assume $a_{1}>a_{2}>\cdots>a_{t}$. Then we associate to the $\beta$-set $X$ the partition $p(X)$ having the non-zero numbers among $a_{i}-(t-i), i=1, \ldots, t$, as parts. For example, $p(\{5,3,0\})=(3,2)$. Note that $p(X)=p\left(X^{+s}\right)$ for all $s \in \mathbb{N}_{0}$.

Let $d \in \mathbb{N}$. We define a $d$-symbol $S=\left(X_{0}, X_{1}, \ldots, X_{d-1}\right)$ as a $d$-tuple of $\beta$-sets. In analogy with $\beta$-sets we define $S^{+s}=\left(X_{0}^{+s}, X_{1}^{+s}, \ldots, X_{d-1}^{+s}\right)$.

In [2], a $d$-symbol is an equivalence class of such $d$-tuples. The equivalence relation is generated by the following operations: 
- cyclic permutation of the $\beta$-sets

- replacing $S$ by $S^{+1}$.

The multiset of all hook lengths in $S$, as defined in [2], only depends on the equivalence class of $S$. The more general definition of hook lengths in symbols which we introduce in Sect. 3 includes those of [2], but they are not independent of the cyclic permutations of the $\beta$-sets.

Let $s \in \mathbb{N}$. We set $[s]=\{0,1, \ldots, s-1\}$. For $n \in \mathbb{N}_{0}$, we define $n_{[s]} \in[s]$ as the remainder of $n$ after division by $s$.

Definition 1.1 Given $d \in \mathbb{N}$ we associate to a $\beta$-set $X$ a $d$-symbol

$$
s_{d}(X)=\left(X_{0}^{(d)}, X_{1}^{(d)}, \ldots, X_{d-1}^{(d)}\right),
$$

where for $j \in[d]$

$$
X_{j}^{(d)}=\left\{k \in \mathbb{N}_{0} \mid j+k d \in X\right\}
$$

Clearly, we have

Lemma 1.2 The map $s_{d}$ is a bijection between the set of all $\beta$-sets and the set of all d-symbols.

Definition 1.3 To an arbitrary $d$-symbol $S$, we associate a partition $p(S)$, defined by

$$
p(S)=p\left(s_{d}^{-1}(S)\right) \text {. }
$$

Definition 1.4 (1) A hook in the $\beta$-set $X$ is a pair $(a, b)$ of nonnegative integers with $a>b$ such that $a \in X$ and $b \notin X$. The set of hooks in $X$ is denoted $H(X)$. If $a-b=\ell$ then $(a, b)$ is called an $\ell$-hook in $X$.

(2) A hook in the $d$-symbol $S=\left(X_{0}, X_{1}, \ldots, X_{d-1}\right)$ is a quadruple $(a, b, i, j)$ of nonnegative integers where $a \geq b, i, j \in[d], a \in X_{i}, b \notin X_{j}$, and in addition if $a=b$ then $i>j$. If $a=b$, we call the hook short and otherwise long. The set of hooks in $S$ is denoted by $H(S)$. If $a-b=\ell$ and $(i-j)_{[d]}=e, e \in[d]$ then $(a, b, i, j)$ is called an $(\ell, e)$-hook in $S$.

Note (i) To avoid confusion we want to point out that since we will be dealing with generalized hook lengths below, an $\ell$-hook will not be the same as a hook of length $\ell$.

(ii) In [2] only long hooks are considered in symbols, since short hooks give only trivial contributions to the unipotent degrees. The inclusion of short hooks makes our later arguments much simpler, and the short hooks will then be dealt with separately whenever necessary.

Lemma 1.5 (1) There is a canonical bijection $\mathfrak{h}_{X}$ between the set $H(X)$ of hooks in a $\beta$-set $X$ and the set $H(p(X))$ of hooks in the partition $p(X)$. Thereby an $\ell$-hook in $H(X)$ is mapped to an $\ell$-hook in the partition $H(p(X))$.

(2) There is a canonical bijection $\mathfrak{h}_{X, d}$ between the set $H(X)$ of hooks in a $\beta$-set $X$ and the set $H(S)$ of hooks in the associated $d$-symbol $S=s_{d}(X)$. 
(3) There is a canonical bijection $\mathfrak{h}_{S}$ between the set $H(S)$ of hooks in a d-symbol $S$ and the set $H(p(S))$ of hooks in the partition $p(S)$.

Proof Let $X=\left\{a_{1}, a_{2}, \ldots, a_{t}\right\}$. (1) If $(a, b) \in H(X), a-b=\ell$ and $a=a_{i}$, then the partition $p(X)$ has an $\ell$-hook in the $i$ th row. The map $\mathfrak{h}_{X}$ sending $(a, b)$ to this hook is a bijection; this is well known, see [1, Sect. 2.7] or [4, Sect. 1] for more details.

(2) The map $\mathfrak{h}_{X, d}$ which maps $(a, b) \in H(X)$ to $\left(a^{\prime}, b^{\prime}, i, j\right) \in H(S)$ where $a=a^{\prime} d+i, b=b^{\prime} d+j$ with $i, j \in[d]$, is obviously a bijection between $H(X)$ and $H(S)=H\left(s_{d}(X)\right)$.

(3) By Definition 1.3, $p(S)=p\left(s_{d}^{-1}(S)\right)$. Put $\mathfrak{h}_{S}=\mathfrak{h}_{X} \circ \mathfrak{h}_{X, d}^{-1}$, where $X=$ $s_{d}^{-1}(S)$.

Definition 1.6 (1) Let $X$ be a $\beta$-set and $z=(a, b) \in H(X)$. If $X^{\prime}=(X \backslash\{a\}) \cup\{b\}$ we say that $X^{\prime}$ is obtained by removing the hook $z$ from $X$.

(2) Let $S=\left(X_{0}, \ldots, X_{d-1}\right)$ be a $d$-symbol and $z=(a, b, i, j) \in H(S)$. If $i \neq j$, we set $X_{i}^{\prime}=X_{i} \backslash\{a\}, X_{j}^{\prime}=X_{j} \cup\{b\}$, for $i=j$ we set $X_{i}^{\prime}=\left(X_{i} \backslash\{a\}\right) \cup\{b\}$, and we set $X_{k}^{\prime}=X_{k}$ for all $k \neq i, j$. Then we say that $S^{\prime}=\left(X_{0}^{\prime}, \ldots, X_{d-1}^{\prime}\right)$ is obtained by removing the hook $z$ from $S$.

Remark 1.7 If we keep removing $\ell$-hooks from a $\beta$-set $X$ for a fixed $\ell$ we eventually reach a $\beta$-set with no $\ell$-hooks left. This is the $\ell$-core of $X$, denoted $C_{\ell}(X)$. By an abacus argument this is well-defined [1, 2.7.16]. A similar statement is true for $(\ell, e)$ hooks in a symbol $[2,3.4]$. We return to this in Sect. 5 .

We call a $d$-symbol $S=\left(X_{0}, \ldots, X_{d-1}\right)$ balanced if $\left|X_{0}\right|=\left|X_{1}\right|=\cdots=\left|X_{d-1}\right|$, and if in addition there is an $i \in[d]$ such that $0 \notin X_{i}$.

To a $d$-tuple $\left(\kappa_{0}, \kappa_{1}, \ldots, \kappa_{d-1}\right)$ of partitions we associate a balanced $d$-symbol as follows. If $r$ is the maximal length (i.e., number of parts) of the partitions $\kappa_{i}$, we may choose a $\beta$-set $Y_{i}$ of cardinality $r$ for each partition $\kappa_{i}$. Then $0 \notin Y_{j}$ whenever $\kappa_{j}$ has length $r$ because then $Y_{j}$ is just the set of first column hook lengths of $\kappa_{j}$. Thus

$$
t_{d}\left(\kappa_{0}, \kappa_{1}, \ldots, \kappa_{d-1}\right)=\left(Y_{0}, Y_{1}, \ldots, Y_{d-1}\right)
$$

is a well-defined balanced $d$-symbol for the $d$-tuple of partitions. On the other hand, if $S=\left(X_{0}, \ldots, X_{d-1}\right)$ is balanced, then $t_{d}\left(p\left(X_{0}\right), \ldots, p\left(X_{d-1}\right)\right)=\left(X_{0}, \ldots, X_{d-1}\right)$.

We have shown:

Lemma 1.8 There is a bijection $t_{d}$ between the set of $d$-tuples of partitions and the set of balanced d-symbols.

Definition 1.9 (1) The balanced quotient $Q(S)$ of an arbitrary $d$-symbol $S=$ $\left(X_{0}, X_{1}, \ldots, X_{d-1}\right)$ is defined as the balanced $d$-symbol

$$
Q(S)=t_{d}\left(p\left(X_{0}\right), p\left(X_{1}\right), \ldots, p\left(X_{d-1}\right)\right) .
$$

We call $q(S)=p(Q(S))$ the quotient partition of $S$. 
(2) The core $C(S)$ of an arbitrary $d$-symbol $S=\left(X_{0}, X_{1}, \ldots, X_{d-1}\right)$ is defined as the $d$-symbol

$$
C(S)=\left(\left[x_{0}\right],\left[x_{1}\right], \ldots,\left[x_{d-1}\right]\right),
$$

where $x_{i}=\left|X_{i}\right|$ for $i \in[d]$. We call $c(S)=p(C(S))$ the core partition of $S$.

Remark 1.10 (A remark on notation) The core $C(S)$ of a $d$-symbol $S$ is really its $(1,0)$-core (see Remark 1.7) and we will consider $Q(S)$ as the $(1,0)$-quotient of $S$. This will be generalized in Sect. 5 .

Remark 1.11 We may recover a $d$-symbol $S=\left(X_{0}, X_{1}, \ldots, X_{d-1}\right)$ from its balanced quotient $Q(S)=\left(Y_{0}, Y_{1}, \ldots, Y_{d-1}\right)$ together with its core $C(S)=\left(Z_{0}, Z_{1}, \ldots, Z_{d-1}\right)$. Indeed, for $i \in[d] X_{i}$ must be the $\beta$-set of cardinality $\left|Z_{i}\right|$ for the partition $p\left(Y_{i}\right)$.

Definition 1.12 Let $X$ be a $\beta$-set. Then the $d$-quotient partition of $X$ is defined as $q_{d}(X)=q\left(s_{d}(X)\right)$ and the $d$-core partition of $X$ is defined as $c_{d}(X)=c\left(s_{d}(X)\right)$.

With notation as in Definition 1.1 we define the $d$-quotient of $X$ as

$$
Q_{d}(X)=s_{d}^{-1}\left(t_{d}\left(p\left(X_{0}^{(d)}\right), p\left(X_{1}^{(d)}\right), \ldots, p\left(X_{d-1}^{(d)}\right)\right)\right) .
$$

Remark 1.13 If we put the elements of a $\beta$-set $X$ as beads on the $d$-abacus, and if $X_{j}^{(d)}$ is as in Definition 1.1, then the results of [1, Sect. 2.7] or [4, Sect. 1] show the following:

- The $d$-core partition $c_{d}(X)$ of $X$ is also the $d$-core $\lambda_{(d)}$ of the partition $\lambda=p(X)$ and thus it stays the same when we replace $X$ by $X^{+s}$.

- We have $c_{d}(X)=p\left(C_{d}(X)\right)$.

- The $d$-quotient partition $q_{d}(X)$ of $X$ must have an empty $d$-core. It may change if we replace $X$ by $X^{+1}$. However $X$ and $X^{+d}$ have the same $d$-quotient partition.

- We have $q_{d}(X)=p\left(Q_{d}(X)\right)$ and $s_{d}\left(Q_{d}(X)\right)=Q\left(s_{d}(X)\right)$.

- If $t \in[d]$ is chosen such that $|X|+t$ is divisible by $d$ then

$$
\left(p\left(X_{t}^{(d)}\right), p\left(X_{t+1}^{(d)}\right), \ldots, p\left(X_{t+d-1}^{(d)}\right)\right)
$$

is the $d$-quotient of the partition $p(X)[1,2.7 .29]$. Here the subscripts are to be read modulo $d$.

Lemma 1.14 Let $X$ be a $\beta$-set, $d \in \mathbb{N}$. Then

$$
|p(X)|=\left|q_{d}(X)\right|+\left|c_{d}(X)\right| .
$$

Proof Using the first and last statement in Remark 1.13 we get

$$
|p(X)|=d\left(\left|p\left(X_{0}^{(d)}\right)\right|+\left|p\left(X_{1}^{(d)}\right)\right|+\cdots+\left|p\left(X_{d-1}^{(d)}\right)\right|\right)+\left|c_{d}(X)\right|
$$

(see e.g. [4, (3.6)(ii)]). If we apply this result to the $d$-quotient of $X$,

$$
Q_{d}(X)=s_{d}^{-1}\left(t_{d}\left(p\left(X_{0}^{(d)}\right), p\left(X_{1}^{(d)}\right), \ldots, p\left(X_{d-1}^{(d)}\right)\right)\right),
$$


and the partition $q_{d}(X)=p\left(Q_{d}(X)\right)$ (which has an empty $d$-core) we get $\left|q_{d}(X)\right|=$ $\left|p\left(Q_{d}(X)\right)\right|=d\left(\left|p\left(X_{0}^{(d)}\right)\right|+\left|p\left(X_{1}^{(d)}\right)\right|+\cdots+\left|p\left(X_{d-1}^{(d)}\right)\right|\right)$. The result follows.

The following diagram illustrates the connection between a $\beta$-set $X$, its associated $d$-symbol $S=s_{d}(X)$, their cores and quotients and the associated partitions via the partition map $p$ :

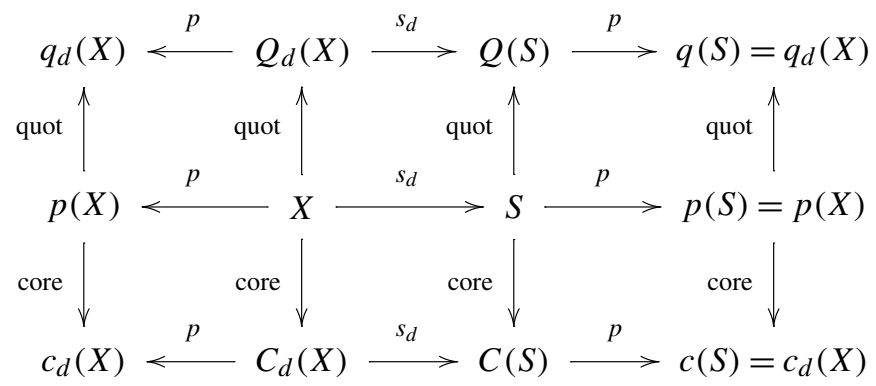

Here is an example:

Example 1.15 Consider the $\beta$-set $X=\{11,8,6,2,0\}$ for the partition $p(X)=\lambda=$ $(7,5,4,1)$ of 17 . Let $d=3$. Letting boldface entries indicate the beads on the abacus we see that the 3 -abacus representation for $X$ is

$\begin{array}{ccc}\mathbf{0} & 1 & \mathbf{2} \\ 3 & 4 & 5 \\ \mathbf{6} & 7 & \mathbf{8} \\ 9 & 10 & \mathbf{1 1}\end{array}$

This corresponds to $S=s_{3}(X)=(\{2,0\}, \emptyset,\{3,2,0\})$ (giving the levels of the beads). We get the balanced quotient

$$
Q(S)=t_{3}((1),(0),(1,1))=(\{2,0\},\{1,0\},\{2,1\}) .
$$

Below on the left, we see its 3-abacus representation and on the right, the 3-abacus representation of the $\beta$-set corresponding to $Q(S)$

\begin{tabular}{|c|c|c|c|c|c|c|}
\hline \multirow{4}{*}{$Q(S)$} & 0 & 0 & 0 & \multirow{3}{*}{$Q_{3}(X)$} & 0 & 1 \\
\hline & 1 & 1 & 1 & & 3 & 4 \\
\hline & 2 & 2 & 2 & & 6 & 7 \\
\hline & 3 & 3 & 3 & & 9 & 10 \\
\hline
\end{tabular}

That is,

$$
Q_{3}(X)=s_{3}^{-1}(Q(S))=\{8,6,5,4,1,0\},
$$

giving the 3-quotient partition

$$
q_{3}(X)=(3,2,2,2) .
$$


The cardinalities of the $\beta$-sets in $S$ give the core symbol

$$
C(S)=(\{1,0\}, \emptyset,\{2,1,0\}) .
$$

The corresponding $\beta$-set (and by construction also the 3-core of $X$ ) is

$$
C_{3}(X)=\{8,5,3,2,0\},
$$

and thus the corresponding 3-core partition is

$$
c_{3}(X)=(4,2,1,1) .
$$

Note that $\left|q_{3}(X)\right|+\left|c_{3}(X)\right|=9+8=17=|p(X)|$, illustrating the previous lemma.

Lemma 1.16 Let $X$ be a $\beta$-set and $S=s_{d}(X)$ be the associated $d$-symbol. We have:

(1) $|p(X)|=|H(p(X))|=|H(X)|=|H(S)|$

(2) $\left|q_{d}(X)\right|=\left|H\left(q_{d}(X)\right)\right|=\left|H\left(Q_{d}(X)\right)\right|=|H(Q(S))|$

(3) $\left|c_{d}(X)\right|=\left|H\left(c_{d}(X)\right)\right|=\left|H\left(C_{d}(X)\right)\right|=|H(C(S))|$

and in addition

$$
\text { (4) }|H(S)|=|H(Q(S))|+|H(C(S))| \text {. }
$$

Proof (1)-(3) are trivial using the bijections in Lemma 1.5 and then (4) follows from Lemma 1.14.

\section{Correspondences between sets of hooks}

In this section we fix the following notation. Let $S=\left(X_{0}, X_{1}, \ldots, X_{d-1}\right)$ be a $d$ symbol with balanced quotient symbol $Q=Q(S)=\left(Y_{0}, Y_{1}, \ldots, Y_{d-1}\right)$ and core symbol $C=C(S)=\left(Z_{0}, Z_{1}, \ldots, Z_{d-1}\right)$.

We would like to describe some well-behaved correspondences (called universal bijections) between the set $H(S)$ and the union of the sets $H(Q)$ and $H(C)$ (see Lemma 1.16(4)).

We decompose the set of hooks $H(S)$ into disjoint subsets according to the position of the hooks. For this, define

$$
H_{i j}(S)=\{(a, b, i, j) \mid(a, b, i, j) \in H(S)\}, \quad H_{i}(S)=H_{i i}(S) .
$$

Then we have

$$
H(S)=\left(\bigcup_{i \in[d]} H_{i}(S)\right) \cup\left(\bigcup_{i, j \in[d], i \neq j} H_{i j}(S)\right) .
$$

Also, we set $H_{\{i j\}}(S)=H_{i j}(S) \cup H_{j i}(S)$. 
We want to split $H_{i j}(S)$ further according to the differences $a-b$, i.e., for $\ell \geq 0$ we define

$$
H_{i j}^{\ell}(S)=\left\{(a, b, i, j) \in H_{i j}(S) \mid a-b=\ell\right\}, \quad H_{i}^{\ell}(S)=H_{i i}^{\ell}(S) .
$$

We have the following easy observation:

Lemma 2.1 Let $i, j \in[d]$. Then we have

$$
\left|H_{i j}^{\ell}(S)\right|= \begin{cases}\left|X_{i}\right|-\left|X_{i} \cap X_{j}^{+\ell}\right| & \text { if } \ell>0, \text { or } \ell=0 \text { and } i>j, \\ 0 & \text { if } \ell=0 \text { and } i \leq j .\end{cases}
$$

Toward our key result, we proceed to describe a correspondence between the set $H_{\{i j\}}(S)$ and the multiset union of the sets $H_{\{i j\}}(Q)$ and $H_{\{i j\}}(C)$, for any given $i, j$.

Note that for each $i \in[d]$ and each $\ell \geq 0$ there is a bijection $H_{i}^{\ell}(S) \rightarrow H_{i}^{\ell}(Q)$ because $p\left(X_{i}\right)=p\left(Y_{i}\right)$, and $H_{i}^{\ell}(C)=\emptyset$.

Thus it suffices to consider the situation where $i \neq j$. We may assume that $i$ and $j$ are such that $\Delta=\left|X_{i}\right|-\left|X_{j}\right| \geq 0$.

For $\Delta=0$ we clearly have $H_{\{i j\}}(S)=H_{\{i j\}}(Q)$ and $H_{\{i j\}}(C)=\emptyset$. So now we consider the case $\Delta>0$. We may assume that $X_{i}=Y_{i}, Y_{j}=X_{j}^{+\Delta}, Z_{i}=[\Delta]$ and $Z_{j}=\emptyset$.

Note that for $1 \leq \ell \leq \Delta, H_{i j}^{\ell}(C)$ has cardinality $\Delta-\ell$. Also $H_{i j}^{0}(C)$ has cardinality 0 if $i<j$ and cardinality $\Delta$ if $j<i$. Clearly, $H_{i j}^{\ell}(C)=\emptyset$ if $\ell>\Delta$, and $H_{j i}^{\ell}(C)=\emptyset$.

First, let $\ell>\Delta$. The conditions $X_{i}=Y_{i}, X_{j}^{+\Delta}=Y_{j}$ imply that we have bijections

$$
\begin{aligned}
& H_{j i}^{\ell-\Delta}(S) \rightarrow H_{j i}^{\ell}(Q), \quad(a, b, j, i) \mapsto(a+\Delta, b, j, i), \\
& H_{i j}^{\ell}(S) \rightarrow H_{i j}^{\ell-\Delta}(Q), \quad(a, b, i, j) \mapsto(a, b+\Delta, i, j) .
\end{aligned}
$$

Next we consider $H_{j i}^{\ell}(Q)$ and $H_{i j}^{\ell}(S)$ for $0<\ell<\Delta$. By Lemma 2.1 we obtain

$$
\left|H_{j i}^{\ell}(Q)\right|=\left|X_{j}\right|+\Delta-\left|X_{j}^{+\Delta} \cap X_{i}^{+\ell}\right|=\left|X_{j}\right|+(\Delta-\ell)-\left|X_{j}^{+(\Delta-\ell)} \cap X_{i}\right|
$$

and then

$$
\left|H_{i j}^{\Delta-\ell}(S)\right|=\left|X_{i}\right|-\left|X_{i} \cap X_{j}^{+(\Delta-\ell)}\right|=\left|H_{j i}^{\ell}(Q)\right|+\ell .
$$

Replacing $\ell$ by $\Delta-\ell$ we have for $0<\ell<\Delta$ :

$$
\left|H_{i j}^{\ell}(S)\right|=\left|H_{j i}^{\Delta-\ell}(Q)\right|+\left|H_{i j}^{\ell}(C)\right| .
$$

It remains to consider the contributions of the sets $H_{i j}^{\Delta}(S), H_{i j}^{0}(S), H_{j i}^{0}(S)$ toward $H_{\{i j\}}(S)$, and of $H_{j i}^{\Delta}(Q), H_{j i}^{0}(Q), H_{i j}^{0}(Q)$ toward $H_{\{i j\}}(Q)$, and take the contribution from $H_{i j}^{0}(C)$ into account. We use again Lemma 2.1 and keep in mind that $X_{j}^{+\Delta}=Y_{j}$. Thus we have

$\left|H_{i j}^{\Delta}(S)\right|=\left|X_{i}\right|-\left|X_{i} \cap Y_{j}\right|, \quad\left|H_{j i}^{\Delta}(Q)\right|=\left|Y_{j}\right|-\left|Y_{j} \cap Y_{i}^{+\Delta}\right|=\left|X_{j}\right|-\left|X_{j} \cap Y_{i}\right|$. 
Furthermore, for $i>j$ we have

$$
\begin{aligned}
& \left|H_{i j}^{0}(S)\right|=\left|X_{i}\right|-\left|X_{i} \cap X_{j}\right|, \quad\left|H_{j i}^{0}(S)\right|=0, \\
& \left|H_{j i}^{0}(Q)\right|=0, \quad\left|H_{i j}^{0}(Q)\right|=\left|Y_{i}\right|-\left|Y_{i} \cap Y_{j}\right|, \quad\left|H_{i j}^{0}(C)\right|=\Delta,
\end{aligned}
$$

and for $i<j$ we have

$$
\begin{aligned}
& \left|H_{i j}^{0}(S)\right|=0, \quad\left|H_{j i}^{0}(S)\right|=\left|X_{j}\right|-\left|X_{j} \cap X_{i}\right|, \\
& \left|H_{j i}^{0}(Q)\right|=\left|Y_{j}\right|-\left|Y_{j} \cap Y_{i}\right|, \quad\left|H_{i j}^{0}(Q)\right|=0, \quad\left|H_{i j}^{0}(C)\right|=0 .
\end{aligned}
$$

Let $k=\max (i, j)$; using that $X_{i}=Y_{i}$ and $\left|Y_{j}\right|=\left|Y_{i}\right|=\left|X_{j}\right|+\Delta$, we see that in both cases the terms add up:

$$
\begin{aligned}
\left|H_{i j}^{\Delta}(S)\right|+\left|H_{\{i j\}}^{0}(S)\right| & =\left|X_{i}\right|-\left|X_{i} \cap Y_{j}\right|+\left|X_{k}\right|-\left|X_{i} \cap X_{j}\right| \\
& =\left|X_{j}\right|-\left|Y_{i} \cap X_{j}\right|+\left|X_{k}\right|+\Delta-\left|Y_{i} \cap Y_{j}\right| \\
& =\left|H_{j i}^{\Delta}(Q)\right|+\left|H_{\{i j\}}^{0}(Q)\right|+\left|H_{i j}^{0}(C)\right| .
\end{aligned}
$$

We have shown the following key result:

Proposition 2.2 Let $S=\left(X_{0}, \ldots, X_{d-1}\right)$ be a d-symbol with balanced quotient symbol $Q(S)=Q$ and core symbol $C(S)=C$. Let $i \neq j \in[d]$, and set $\Delta=\left|X_{i}\right|-\left|X_{j}\right|$. When $\Delta>0$, we have the following equalities:

- For all $\ell>\Delta:\left|H_{i j}^{\ell}(S)\right|=\left|H_{i j}^{\ell-\Delta}(Q)\right|$.

- For all $\ell>\Delta:\left|H_{j i}^{\ell-\Delta}(S)\right|=\left|H_{j i}^{\ell}(Q)\right|$.

- For all $0<\ell<\Delta:\left|H_{i j}^{\ell}(S)\right|=\left|H_{j i}^{\Delta-\ell}(Q)\right|+\left|H_{i j}^{\ell}(C)\right|$.

- $F o r \ell=\Delta:\left|H_{i j}^{\Delta}(S)\right|= \begin{cases}\left|H_{i j}^{0}(Q)\right|=\left|H_{\{i j\}}^{0}(Q)\right| & \text { if } i>j, \\ \left|H_{j i}^{0}(Q)\right|=\left|H_{\{i j\}}^{0}(Q)\right| & \text { if } i<j .\end{cases}$

- $F o r \ell=0:\left|H_{j i}^{\Delta}(Q)\right|+\left|H_{i j}^{0}(C)\right|= \begin{cases}\left|H_{i j}^{0}(S)\right|=\left|H_{\{i j\}}^{0}(S)\right| & \text { if } i>j, \\ \left|H_{j i}^{0}(S)\right|=\left|H_{\{i j\}}^{0}(S)\right| & \text { if } i<j .\end{cases}$

- $\left|H_{i j}^{\Delta}(S)\right|+\left|H_{\{i j\}}^{0}(S)\right|=\left|H_{j i}^{\Delta}(Q)\right|+\left|H_{\{i j\}}^{0}(Q)\right|+\left|H_{i j}^{0}(C)\right|$.

When $\Delta=0$, we have

- $\left|H_{i j}^{\ell}(S)\right|=\left|H_{i j}^{\ell}(Q)\right|, H_{i j}^{\ell}(C)=\emptyset$, for all $\ell \geq 0$.

Using all the correspondences behind the equalities established so far we find bijective correspondences (between multisets)

$$
H_{\{i j\}}(S) \rightarrow H_{\{i j\}}(Q) \cup H_{\{i j\}}(C)
$$

for all $i, j \in[d]$, and we may glue these together to set up a universal bijection

$$
\omega_{S}: H(S) \rightarrow H(Q) \cup H(C) .
$$


Remark 2.3 Let us specify the properties of a universal bijection $\omega_{S}$ very explicitly. Let $i \neq j \in[d]$ be chosen such that $\Delta=\left|X_{i}\right|-\left|X_{j}\right| \geq 0$.

(i) For positive $\ell$ we have:

(1) For all $\ell>\Delta \geq 0$ : $\omega_{S}\left(H_{i j}^{\ell}(S)\right)=H_{i j}^{\ell-\Delta}(Q)$.

(2) For all $\ell>\Delta \geq 0$ : $\omega_{S}\left(H_{j i}^{\ell-\Delta}(S)\right)=H_{j i}^{\ell}(Q)$.

(3) For $\ell=\Delta>0$ : $\omega_{S}\left(H_{i j}^{\Delta}(S)\right)= \begin{cases}H_{i j}^{0}(Q) & \text { if } i>j, \\ H_{j i}^{0}(Q) & \text { if } i<j .\end{cases}$

(4) For all $0<\ell<\Delta: \omega_{S}\left(H_{i j}^{\ell}(S)\right)=H_{j i}^{\Delta-\ell}(Q) \cup H_{i j}^{\ell}(C)$.

(5) For all $\ell: \omega_{S}\left(H_{i}^{\ell}(S)\right)=H_{i}^{\ell}(Q)$.

(ii) For $\ell=0$, we have the following.

For $\Delta>0$ :

(1) $H_{j i}^{\Delta}(Q) \cup H_{i j}^{0}(C)= \begin{cases}\omega_{S}\left(H_{i j}^{0}(S)\right)=\omega_{S}\left(H_{\{i j\}}^{0}(S)\right) & \text { if } i>j, \\ \omega_{S}\left(H_{j i}^{0}(S)\right)=\omega_{S}\left(H_{\{i j\}}^{0}(S)\right) & \text { if } i<j .\end{cases}$

(2) $\omega_{S}\left(H_{i}^{0}(S)\right)=H_{i}^{0}(Q)$.

For $\Delta=0$ :

(3) $\omega_{S}\left(H_{i j}^{0}(S)\right)=H_{i j}^{0}(Q)$.

Of course such a universal bijection is by no means unique and apparently cannot be made "canonical". The important fact for this bijection is the relation between the $\ell$ 's in corresponding hooks.

\section{Generalized hook length functions}

We now want to associate lengths to the hooks in $H(S)$, where $S$ is a $d$-symbol. We define our (generalized) hook length function on the set

$$
H=\left\{(a, b, i, j) \in \mathbb{N}_{0}^{2} \times[d]^{2} \mid a \geq b \text { and } i>j \text { if } a=b\right\} .
$$

In general the lengths may be arbitrary real numbers, i.e., we have a (generalized) hook length function $h: H \rightarrow \mathbb{R}$. However, we only want to consider functions $h$ such that

the value $h(a, b, i, j)$ depends only on $\ell=a-b, i$ and $j$.

This guarantees that the multiset $\mathcal{H}(S)$ of all $h(z), z \in H(S)$, coincides with $\mathcal{H}\left(S^{+s}\right)$ for all $s \in \mathbb{N}_{0}$. Indeed, $(a, b, i, j) \in H(S)$ if and only if $(a+s, b+s, i, j) \in H\left(S^{+s}\right)$, and the $h$-value for these hooks will be the same.

We set

$$
H_{i j}=\{(a, b, i, j) \mid(a, b, i, j) \in H\}, \quad H_{i j}^{\ell}=\left\{(a, b, i, j) \in H_{i j} \mid a-b=\ell\right\}
$$

and then the hook length functions $h$ that we will consider will be constant on $H_{i j}^{\ell}$. 
We now describe some hook length functions of interest for $d$-symbols. For a $(d+1)$-tuple $\delta=\left(c_{0}, c_{1}, \ldots, c_{d-1} ; k\right)$ of real numbers, with $k \geq 0$, we define the $\delta$-length of $(a, b, i, j) \in H$ as

$$
h^{\delta}(a, b, i, j)=k(a-b)+c_{i}-c_{j} .
$$

We call $\delta$ a $d$-hook data tuple. For any $d$-symbol $S$, we let $\mathcal{H}^{\delta}(S)$ be the multiset of all $h^{\delta}(a, b, i, j),(a, b, i, j) \in H(S)$, and $\mathcal{H}_{i j}^{\delta}(S)$ be the multiset of all $h^{\delta}(a, b, i, j)$, $(a, b, i, j) \in H_{i j}(S)$.

Remark 3.1 Some special choices of $d$-hook data tuples will be particularly important in the next sections.

- If we choose $\delta^{o}=(0,0, \ldots, 0 ; 1)$ then the $\delta$-length of long hooks in $S$ coincides with the length defined in [2, p. 782], and the short hooks have $\delta^{o}$-length 0 . We call $\delta^{o}=(0,0, \ldots, 0 ; 1)$ the minimal d-hook data tuple.

- If we choose $\delta^{*}=(0,1, \ldots, d-1 ; d)$ then in the notation of Lemma 1.5 the usual hook length $a-b$ of $(a, b)$ in $H(X)$ equals the $\delta^{*}$-length of the corresponding hook $\mathfrak{h}_{X, d}(a, b)$ in $H(S)$. We call $\delta^{*}=(0,1, \ldots, d-1 ; d)$ the partition d-hook data tuple.

As before, we let $S=\left(X_{0}, X_{1}, \ldots, X_{d-1}\right)$ be a $d$-symbol with balanced quotient

$$
Q=Q(S)=\left(Y_{0}, Y_{1}, \ldots, Y_{d-1}\right)
$$

and core

$$
C=C(S)=\left(\left[x_{0}\right],\left[x_{1}\right], \ldots,\left[x_{d-1}\right]\right),
$$

where $x_{i}=\left|X_{i}\right|$. We have set up above a universal bijection

$$
\omega_{S}: H(S) \rightarrow H(Q) \cup H(C),
$$

with properties specified in Remark 2.3.

Let $\delta=\left(c_{0}, c_{1}, \ldots, c_{d-1} ; k\right)$ be an arbitrary $d$-hook data tuple. We define

$$
\delta_{S}:=\left(c_{0}+x_{0} k, c_{1}+x_{1} k, \ldots, c_{d-1}+x_{d-1} k ; k\right) .
$$

This is a new $d$-hook data tuple which depends on the core of $S$.

We want to modify $h=h^{\delta}$ to a new length function $\bar{h}=\bar{h}^{\delta_{S}}$ such that for $z \in H(S)$ we have

$$
h^{\delta}(z)= \begin{cases}\bar{h}^{\delta_{S}}\left(\omega_{S}(z)\right) & \text { if } \omega_{S}(z) \in H(Q), \\ h^{\delta}\left(\omega_{S}(z)\right) & \text { if } \omega_{S}(z) \in H(C) .\end{cases}
$$

If this is done we immediately have

$$
\mathcal{H}^{\delta}(S)=\overline{\mathcal{H}}^{\delta_{S}}(Q) \cup \mathcal{H}^{\delta}(C),
$$

where $\overline{\mathcal{H}}^{\delta_{S}}(Q)$ is the multiset of all $\bar{h}^{\delta_{S}}(z), z \in H(Q)$. 
We proceed to define $\bar{h}^{\delta_{S}}$. Apart from a sign, $\bar{h}^{\delta_{S}}$ is just $h^{\delta_{S}}$, with $\delta_{S}$ as above. Let us describe the sign modification of $h^{\delta_{S}}$ on $H_{\{i j\}}=H_{i j} \cup H_{j i}$.

We set $H_{i j}^{>m}=\bigcup_{\ell>m} H_{i j}^{\ell}$, and use similar notation for the condition $\geq m$ and for subsets of $H_{j i}$.

We assume that $i, j \in[d]$ are such that $\Delta=x_{i}-x_{j} \geq 0$. Then for $z \in H_{\{i j\}}^{\ell}$ we define

$$
\bar{h}^{\delta_{S}}(z)= \begin{cases}h^{\delta_{S}}(z) & \text { if } z \in H_{i j} \cup H_{j i}^{>\Delta}, \text { or } z \in H_{j i}^{\Delta} \text { if } i<j \\ -h^{\delta_{S}}(z) & \text { otherwise }\end{cases}
$$

Theorem 3.2 Let $\omega_{S}$ be a universal bijection. Let $h=h^{\delta}$ and $\bar{h}=\bar{h}^{\delta_{S}}$ be as defined above. Then for $z \in H(S)$ we have

$$
h^{\delta}(z)= \begin{cases}\bar{h}^{\delta_{S}}\left(\omega_{S}(z)\right) & \text { if } \omega_{S}(z) \in H(Q), \\ h^{\delta}\left(\omega_{S}(z)\right) & \text { if } \omega_{S}(z) \in H(C) .\end{cases}
$$

Proof Let $i, j \in[d]$. As above, we assume that $i, j$ are chosen such that $\Delta:=x_{i}-$ $x_{j} \geq 0$. We refer to Remark 2.3 for the properties of $\omega_{S}$ used below. For $i=j$, the claim clearly holds, as $\omega_{S}\left(H_{i}^{\ell}(S)\right)=H_{i}^{\ell}(Q)$ for all $\ell \geq 0$. Thus we assume now that $i \neq j$.

First assume $\Delta=0$. Then $\omega_{S}\left(H_{i j}^{\ell}(S)\right)=H_{i j}^{\ell}(Q)$ for all $\ell \geq 0$, and by definition we have for $z \in H_{i j}^{\ell}(S)$ (and analogously for $z \in H_{j i}^{\ell}(S)$ ):

$$
h^{\delta}(z)=\ell k+c_{i}-c_{j}=h^{\delta_{S}}\left(\omega_{S}(z)\right)=\bar{h}^{\delta_{S}}\left(\omega_{S}(z)\right) .
$$

Now assume $\Delta>0$. First consider the case $\ell>\Delta$. Take $z \in H_{i j}^{\ell}(S)$. Then we know that $\omega_{S}(z) \in H_{i j}^{\ell-\Delta}(Q)$ and by definition

$$
\begin{aligned}
\bar{h}^{\delta_{S}}\left(\omega_{S}(z)\right) & =h^{\delta_{S}}\left(\omega_{S}(z)\right)=(\ell-\Delta) k+\left(c_{i}+x_{i} k\right)-\left(c_{j}+x_{j} k\right) \\
& =\ell k+c_{i}-c_{j}=h^{\delta}(z) .
\end{aligned}
$$

Also, if $z \in H_{j i}^{\ell-\Delta}(S)$ then $\omega_{S}(z) \in H_{j i}^{\ell}(Q)$ and again

$$
\begin{aligned}
\bar{h}^{\delta_{S}}\left(\omega_{S}(z)\right) & =h^{\delta_{S}}\left(\omega_{S}(z)\right)=\ell k+\left(c_{j}+x_{j} k\right)-\left(c_{i}+x_{i} k\right) \\
& =(\ell-\Delta) k+c_{j}-c_{i}=h^{\delta}(z) .
\end{aligned}
$$

Suppose next that $0<\ell<\Delta$. If $z \in H_{i j}^{\ell}(S)$ then either $\omega_{S}(z) \in H_{j i}^{\Delta-\ell}(Q)$ or $\omega_{S}(z) \in H_{i j}^{\ell}(C)$. In the latter case, clearly $h^{\delta}\left(\omega_{S}(z)\right)=h^{\delta}(z)$. In the former case we compute

$$
\begin{aligned}
\bar{h}^{\delta_{S}}\left(\omega_{S}(z)\right) & =-h^{\delta_{S}}\left(\omega_{S}(z)\right)=(\ell-\Delta) k-\left(c_{j}+x_{j} k\right)+\left(c_{i}+x_{i} k\right) \\
& =\ell k-c_{j}+c_{i}=h^{\delta}(z) .
\end{aligned}
$$


If $\ell=0, z \in H_{\{i j\}}^{0}(S)$, we have to distinguish the cases $i>j$ and $i<j$. When $i>j, z \in H_{i j}^{0}(S)$, and $z$ can be mapped to either $\omega_{S}(z) \in H_{j i}^{\Delta}(Q)$ or to $\omega_{S}(z) \in$ $H_{i j}^{0}(C)$. The latter case is clear, and in the former case we compute

$$
\bar{h}^{\delta_{S}}\left(\omega_{S}(z)\right)=-h^{\delta_{S}}\left(\omega_{S}(z)\right)=-\Delta k-\left(c_{j}+x_{j} k\right)+\left(c_{i}+x_{i} k\right)=c_{i}-c_{j}=h^{\delta}(z) .
$$

When $i<j, z \in H_{j i}^{0}(S), z$ is mapped to $\omega_{S}(z) \in H_{j i}^{\Delta}(Q)$, and we compute

$$
\bar{h}^{\delta_{S}}\left(\omega_{S}(z)\right)=h^{\delta_{S}}\left(\omega_{S}(z)\right)=\Delta k+\left(c_{j}+x_{j} k\right)-\left(c_{i}+x_{i} k\right)=c_{j}-c_{i}=h^{\delta}(z) .
$$

Finally, we are in the case $z \in H_{i j}^{\Delta}(S)$. Then $\omega_{S}(z) \in H_{\{i j\}}^{0}(Q)$, and again we have to distinguish the cases $i>j$ and $i<j$. When $i>j, \omega_{S}(z) \in H_{i j}^{0}(Q)$ and we have

$$
\bar{h}^{\delta_{S}}\left(\omega_{S}(z)\right)=h^{\delta_{S}}\left(\omega_{S}(z)\right)=c_{i}+x_{i} k-\left(c_{j}+x_{j} k\right)=\Delta k+c_{i}-c_{j}=h^{\delta}(z) .
$$

When $i<j, \omega_{S}(z) \in H_{j i}^{0}(Q)$ and we have

$$
\bar{h}^{\delta S}\left(\omega_{S}(z)\right)=-h^{\delta_{S}}\left(\omega_{S}(z)\right)=-\left(c_{j}+x_{j} k\right)+c_{i}+x_{i} k=\Delta k+c_{i}-c_{j}=h^{\delta}(z) .
$$

Now we have dealt with all the elements in $H_{\{i j\}}(S)$ and the assertion is proved.

As indicated before, the above theorem has the following consequence, which we will use repeatedly in the following.

Theorem 3.3 Let $S=\left(X_{0}, X_{1}, \ldots, X_{d-1}\right)$ be a $d$-symbol with balanced quotient $Q=Q(S)$ and core $C=C(S)=\left(\left[x_{0}\right],\left[x_{1}\right], \ldots,\left[x_{d-1}\right]\right)$, where $x_{i}=\left|X_{i}\right|$. Let $\delta=$ $\left(c_{0}, c_{1}, \ldots, c_{d-1} ; k\right)$ be a $d$-hook data tuple and $\delta_{S}=\left(c_{0}+x_{0} k, c_{1}+x_{1} k, \ldots, c_{d-1}+\right.$ $\left.x_{d-1} k ; k\right)$. Then we have the multiset equality

$$
\mathcal{H}^{\delta}(S)=\overline{\mathcal{H}}^{\delta_{S}}(Q) \cup \mathcal{H}^{\delta}(C),
$$

where $\overline{\mathcal{H}}^{\delta_{S}}(Q)$ is the multiset of all $\bar{h}^{\delta_{S}}(z), z \in H(Q)$.

In particular we have the multiset inclusion

$$
\mathcal{H}^{\delta}(C) \subseteq \mathcal{H}^{\delta}(S)
$$

For a later application we need the following

Remark 3.4 (Reversal of short hooks) We consider another possible definition of a short hook in a symbol, which we call a "reversed" short hook. Suppose that $S=$ $\left(X_{0}, X_{1}, \ldots, X_{d-1}\right)$. By Definition 1.4 a short hook in $S$ is given by $(a, a, i, j)$ where $a \in X_{i}, a \notin X_{j}$ and $i>j$. Clearly short hooks from $i$ to $j$ are determined by the elements $a \in X_{i} \backslash\left(X_{i} \cap X_{j}\right)$. The reversed short hooks in $S$ are given by $(a, a, i, j)$ where $a \in X_{i}, a \notin X_{j}$ and $i<j$. If $i>j$ and $\delta=\left(c_{1}, \ldots, c_{d-1} ; k\right)$ is a $d$-hook data tuple then the $\delta$-length of a short hook from $i$ to $j$ is $c_{i}-c_{j}$ whereas the $\delta$-length of 
a reversed short hook from $j$ to $i$ is $c_{j}-c_{i}$. We note that these lengths are equal up to a sign.

Whenever $i>j$ then the number of short hooks from $i$ to $j$ is $\left|X_{i} \backslash\left(X_{i} \cap X_{j}\right)\right|$, whereas the number of reversed short hooks from $j$ to $i$ is $\left|X_{j} \backslash\left(X_{i} \cap X_{j}\right)\right|$. If the symbol $S$ is balanced, these numbers are equal, since then $\left|X_{i}\right|=\left|X_{j}\right|$.

\section{Partition data tuples and hooks in partitions}

For any partition $\lambda$ we denote by $\mathcal{H}(\lambda)$ the multiset of (usual) hook lengths in $\lambda$. Let $X$ be any $\beta$-set and $d \in \mathbb{N}$. We let $\mathcal{H}(X)$ be the multiset of hook lengths in $X$ (in the sense of Definition 1.4(1)). Note that all these hook lengths are positive integers. The bijection $\mathfrak{h}_{X}$ from Lemma 1.5(1) preserves hook lengths. Thus we have

Lemma 4.1 Let $X$ be a $\beta$-set for the partition $p(X)$. Then

$$
\mathcal{H}(X)=\mathcal{H}(p(X)) .
$$

Consider the associated $d$-symbol $S=s_{d}(X)$ to $X$. Lemma 1.5 shows that there is a bijection $\mathfrak{h}=\mathfrak{h}_{X, d}$ between $H(X)$ and $H(S)$. If we choose the partition $d$-hook data tuple $\delta^{*}=(0,1, \ldots, d-1 ; d)$ for $S$ then the description of $\mathfrak{h}$ shows that for any hook $z \in H(X)$ we have $h(z)=h^{\delta^{*}}(\mathfrak{h}(z))$ (Remark 3.1).

Thus we obtain

Lemma 4.2 Let $X$ be a $\beta$-set with associated $d$-symbol $S=s_{d}(X)$. Then

$$
\mathcal{H}(X)=\mathcal{H}^{\delta^{*}}(S)
$$

where $\delta^{*}$ is the partition d-hook data tuple.

If we then apply Theorem 3.3 to a $d$-symbol $S$ and the partition $d$-hook data tuple $\delta^{*}$ we get the following result:

Theorem 4.3 Let the $d$-symbol $S=\left(X_{0}, X_{1}, \ldots, X_{d-1}\right)$ have the balanced quotient $Q=Q(S)$ and the core $C=C(S)=\left(\left[x_{0}\right],\left[x_{1}\right], \ldots,\left[x_{d-1}\right]\right)$, where $\left|X_{i}\right|=x_{i}$ for $0 \leq i \leq d-1$. Let the $d$-hook data tuple $\delta_{S}^{*}$ be defined by $\delta_{S}^{*}=\left(x_{0} d, 1+x_{1} d, \ldots\right.$, $\left.(d-1)+x_{d-1} d ; d\right)$. Then

$$
\mathcal{H}^{\delta^{*}}(S)=\mathcal{H}^{\delta^{*}}(C) \cup \operatorname{abs}\left(\mathcal{H}^{\delta^{*}}(Q)\right),
$$

where $\operatorname{abs}\left(\mathcal{H}^{\delta_{S}^{*}}(Q)\right)=\left\{|h| \mid h \in \mathcal{H}^{\delta_{S}^{*}}(Q)\right\}$.

Proof In the notation of Theorem 3.3, an element $\bar{h}^{\delta_{S}^{*}}(z) \in \overline{\mathcal{H}}^{\delta_{S}^{*}}(Q), z \in H(Q)$, has the same absolute value as $h^{\delta_{S}^{*}}(z)$. Since the elements of $\mathcal{H}^{\delta^{*}}(S)$ are positive integers, the result follows.

We may translate Theorem 4.3 into a statement about hooks in partitions; we use the notation introduced in Definitions 1.1 and 1.12. 
Theorem 4.4 Let $d \in \mathbb{N}$ and let $\lambda$ be a partition. Let $X$ be a $\beta$-set for $\lambda$; we set $x_{i}=\left|X_{i}^{(d)}\right|$, for $i \in[d]$, and put $\delta=\left(x_{0} d, 1+x_{1} d, \ldots,(d-1)+x_{d-1} d ; d\right)$. Let $Q=Q\left(s_{d}(X)\right)$, a balanced symbol. Then

$$
\mathcal{H}(\lambda)=\mathcal{H}\left(\lambda_{(d)}\right) \cup \operatorname{abs}\left(\mathcal{H}^{\delta}(Q)\right)
$$

where $\operatorname{abs}\left(\mathcal{H}^{\delta}(Q)\right)=\left\{|h| \mid h \in \mathcal{H}^{\delta}(Q)\right\}$.

Let us add a remark to the theorem.

Remark 4.5 Theorem 4.4 states in particular that the multiset of hook lengths of the $d$-core of a partition is contained in that of the partition. This suggests the following question. Suppose that $\mu$ is obtained from $\lambda$ by removing a number of $d$-hooks; is it then true that $\mathcal{H}(\mu) \subseteq \mathcal{H}(\lambda)$ ? The answer is definitely no, and there are numerous examples for any $d$. For instance, $\mu=(2 d, 1)$ is obtained from $\lambda=\left(2 d, 1^{d+1}\right)$ by removing a $d$-hook, but $2 d+1 \in \mathcal{H}(\mu)$ and $2 d+1 \notin \mathcal{H}(\lambda)$.

For any $d$-symbol $S$ there is a canonical bijection $\mathfrak{h}_{S}$ between $H(S)$ and $H(p(S))$ (Lemma 1.5). This may be applied to the balanced symbol $Q$ of Theorem 4.4. Then $p(Q)$ is the $d$-quotient partition $q_{d}(X)$ of the $\beta$-set $X$ of $\lambda$. The lengths of corresponding hooks differ up to a sign only by a multiple of $d$. Thus $\mathcal{H}^{\delta}(Q)$ may be seen as a multiset of modified hook lengths of the $d$-quotient partition $q_{d}(X)$.

To be more specific we need the $d$-residues of the nodes in a Young diagram. The node $(k, l)$ in row $k$ and column $l$ and the corresponding hook have $(d$-)residue $e=(l-k)_{[d]} \in[d]$. For a partition $\lambda=\left(\ell_{1}, \ldots, \ell_{r}\right)$, the rightmost residue in row $k$ is called the hand $(d-)$ residue and the bottom residue in column $l$ is called the foot $\left(d\right.$-)residue of the $(k, l)$-hook in $\lambda$. If $X=\left\{a_{1}, a_{2}, \ldots, a_{s}\right\}$ is a $\beta$-set for $\lambda$, then $\ell_{k}=a_{k}-(s-k)$. Thus if $|X|=s$ is divisible by $d$, then $a_{k[d]}=\left(\ell_{k}-k\right)_{[d]}$ is the end residue in row $k$ of $\lambda$. Note that if the $(k, l)$-hook has length $m$, then its foot residue is congruent to $a_{k}-m+1$. Thus we have the following:

Lemma 4.6 Let $X$ be a $\beta$-set for $\lambda$ such that $d|| X \mid$. Let $(a, b) \in H(X)$. Then the hand and foot $\left(d\right.$-)residue of the corresponding hook $\mathfrak{h}_{X}(a, b) \in H(\lambda)$ are $a_{[d]}$ and $b_{[d]}+1$, respectively.

We may then reformulate Theorem 4.4, involving a $d$-quotient partition instead of a balanced symbol, and a suitably modified hook length.

Theorem 4.7 Let $d \in \mathbb{N}, \lambda$ a partition, $X$ a $\beta$-set for $\lambda, x_{i}=\left|X_{i}^{(d)}\right|, i \in[d]$. Let $\lambda_{X}^{(d)}=q_{d}(X)$ be the $d$-quotient partition of $X$. For $z \in H\left(\lambda_{X}^{(d)}\right)$, we define a modified hook length as $\bar{h}(z)=h(z)+\left(x_{i}-x_{j}\right) d$, if $z$ has hand and foot $(d-)$ residue $i$ and $j+1$, respectively. We denote by $\overline{\mathcal{H}}\left(\lambda_{X}^{(d)}\right)$ the multiset of all $\bar{h}(z), z \in H\left(\lambda_{X}^{(d)}\right)$. Then

$$
\mathcal{H}(\lambda)=\mathcal{H}\left(\lambda_{(d)}\right) \cup \operatorname{abs}\left(\overline{\mathcal{H}}\left(\lambda_{X}^{(d)}\right)\right),
$$

where $\left.\operatorname{abs}\left(\overline{\mathcal{H}}\left(\lambda_{X}^{(d)}\right)\right)=\left\{|h| \mid h \in \overline{\mathcal{H}}\left(\lambda_{X}^{(d)}\right)\right)\right\}$. 
Proof It suffices to show that if $Q=Q\left(s_{d}(X)\right)$ and $\delta$ are as in Theorem 4.4, then $\mathcal{H}^{\delta}(Q)=\overline{\mathcal{H}}\left(\lambda_{X}^{(d)}\right)$. We have $p(Q)=\lambda_{X}^{(d)}$ and there is a canonical bijection $\mathfrak{h}$ between $H(Q)$ and $H\left(\lambda_{X}^{(d)}\right)$ (Lemma 1.5). In fact, $\mathfrak{h}=\mathfrak{h}_{Q_{d}(X)} \circ \mathfrak{h}_{Q_{d}(X), d}^{-1}$. Note that $Q=$ $s_{d}\left(Q_{d}(X)\right)$, and since $Q$ is balanced, we have $d|| Q_{d}(X) \mid$. Thus if $z \in H\left(\lambda_{X}^{(d)}\right)$ has hand and foot $\left(d\right.$-)residues $i$ and $j+1$, respectively, then by Lemma $4.6 \mathfrak{h}_{Q_{d}(X)}^{-1}$ maps $z$ into a pair $(a, b) \in H\left(Q_{d}(X)\right)$ where $a_{[d]}=i$ and $b_{[d]}=j$. Writing $a=a^{\prime} d+i, b=$ $b^{\prime} d+j$ we have $\mathfrak{h}(z)=\left(a^{\prime}, b^{\prime}, i, j\right)$. Now $h(z)=a-b=\left(a^{\prime}-b^{\prime}\right) d+i-j$, and by the definition of $\delta$ we have $h^{\delta}\left(a^{\prime}, b^{\prime}, i, j\right)=\bar{h}(z)$.

We illustrate Theorem 4.7 by an example.

Example 4.8 (This continues Example 1.15) Consider the $\beta$-set $X=\{11,8,6,2,0\}$ for the partition $p(X)=\lambda=(7,5,4,1)$ of 17 . Let $d=3$. We computed the 3 -quotient partition

$$
\lambda_{X}^{(3)}=q_{3}(X)=(3,2,2,2)
$$

and the 3-core partition

$$
\lambda_{(3)}=c_{3}(X)=(4,2,1,1) .
$$

Note that $|\lambda|=17=9+8=\left|\lambda_{X}^{(3)}\right|+\left|\lambda_{(3)}\right|$. Moreover, the numbers $x_{i}$ of elements in $X$ congruent to $i$ modulo 3 are $(2,0,3)$.

Consider the hook diagrams of $\lambda$ and $\lambda_{(3)}$ where we have marked by boldface eight hook lengths in $\lambda$ which also occur in $\lambda(3)$.

$\begin{array}{ccccccccccccc} & 10 & 8 & \mathbf{7} & 6 & \mathbf{4} & \mathbf{2} & \mathbf{1} & & 7 & 4 & 2 & 1 \\ & 7 & 5 & \mathbf{4} & 3 & \mathbf{1} & & & & 4 & 1 & & \\ & 5 & 3 & \mathbf{2} & 1 & & & & \lambda_{(3)} & 2 & & & \\ & \mathbf{1} & & & & & & & & & & & \end{array}$

The remaining nine hook lengths in $\lambda$ which are not in $\lambda_{(3)}$ are

$$
R=\{1,3,3,5,5,6,7,8,10\}
$$

We obtain these by adjusting the hook lengths of $\lambda_{X}^{(3)}$ by multiples of 3 and changing signs of negative entries. Consider first the 3-residue diagram of $\lambda_{X}^{(3)}$ :

$\begin{array}{lll}0 & 1 & 2 \\ 2 & 0 & \\ 1 & 2 & \\ 0 & 1 & \end{array}$

We add $3 x_{i}$ to hook lengths in rows with end residue $i$ and subtract $3 x_{j}$ from hook lengths in columns with end residue $j+1$. These multiples of 3 are listed in boldface 
in the rows and columns of the hook diagram of $\lambda_{X}^{(3)}$ to the left, and we show the result (before sign change) to the right:

\begin{tabular}{r|rrrrrr} 
& $\mathbf{9}$ & $\mathbf{6}$ & $\mathbf{0}$ & & & \\
\hline $\mathbf{9}$ & 6 & 5 & 1 & 6 & 8 & 10 \\
$\mathbf{6}$ & 4 & 3 & & 1 & 3 & \\
$\mathbf{9}$ & 3 & 2 & & 3 & 5 & \\
$\mathbf{0}$ & 2 & 1 & & -7 & -5 &
\end{tabular}

Changing the sign of -7 and -5 we get exactly the hook lengths in the list $R$.

Whenever $M$ is a multiset of real numbers, we let $\prod M$ denote the product of all the elements in $M$. Thus if $\lambda$ is a partition, then $\prod \mathcal{H}(\lambda)$ is the product of all hook lengths in $\lambda$. Using the notation of Lemmas 4.1, 4.2 we have the following.

Corollary 4.9 $\prod \mathcal{H}(X)=\prod \mathcal{H}(\lambda)=\prod \mathcal{H}^{\delta^{*}}(S)$.

Corollary 4.10 With the notation of Theorem 4.4 we have

$$
\prod \mathcal{H}(\lambda)=\prod \mathcal{H}\left(\lambda_{(d)}\right) \cdot\left|\prod \mathcal{H}^{\delta}(Q)\right|
$$

The celebrated hook formula for the degrees of the irreducible characters of the symmetric group $S_{n}$ may be formulated as follows.

Theorem 4.11 Let $\lambda$ be a partition of $n$, and let $\chi_{\lambda}$ be the irreducible character of $S_{n}$ labeled by $\lambda$. Then

$$
\chi_{\lambda}(1)=\frac{n !}{\prod \mathcal{H}(\lambda)} .
$$

Then Corollary 4.10 may be formulated as follows:

Corollary 4.12 If $\left|\lambda_{(d)}\right|=r$ then with the notation of Theorem 4.4 we have

$$
\chi_{\lambda}(1)=\frac{n !}{r !} \frac{1}{\left|\prod \mathcal{H}^{\delta}(Q)\right|} \chi_{\lambda_{(d)}}(1)
$$

Remark 4.13 Corollary 4.12 is equivalent to a generalization of [3, Theorem 9.1]. In this theorem, $d$ is assumed to be a prime, the $\beta$-set $X$ for $\lambda$ is chosen to be the set of first column hook lengths for $\lambda$, and the short hooks in the balanced symbol $Q$ (which is called $S$ in [3, Theorem 9.1]) are reversed. As pointed out in Remark 3.4 above, the reversal of short hooks does not influence the absolute value of the products $\prod \mathcal{H}^{\delta}(Q)$ of all hook lengths. 


\section{Minimal data tuples and hooks in symbols}

Let $S=\left(X_{0}, X_{1}, \ldots, X_{d-1}\right)$ be a $d$-symbol. Given $\ell \in \mathbb{N}$ and $e \in[d]$ we want to consider the $(\ell, e)$-core and $(\ell, e)$-quotient of $S$. We start by the case $e=0$, where our result Theorem 5.2 is slightly stronger than in the general case, Theorem 5.4. Only the short hooks create a difficulty in the general case.

Cores and quotients of symbols are also considered in [2, Sect. 3] and an analysis closely related to our results is contained in [2, Proof of Theorem 3.14].

First we define $S_{* \ell}$ as the $d \ell$-symbol

$$
S_{* \ell}:=s_{d \ell}\left(s_{d}^{-1}(S)\right) .
$$

Here $s_{d}^{-1}$ transforms the $d$-symbol $S$ into a $\beta$-set $X$ and $S_{* \ell}$ is then the $d \ell$-symbol associated to $X$. Thus $S_{* \ell}$ may be seen as the "splitting of $S$ into $\ell$ pieces".

By Definition 1.9 the $d \ell$-symbol $S_{* \ell}$ has a balanced quotient which we call the balanced $\ell$-quotient of $S$ :

$$
Q_{\ell}(S):=Q\left(S_{* \ell}\right)
$$

The $d \ell$-symbol $S_{* \ell}$ also has a core $C\left(S_{* \ell}\right)$. By Lemma 1.5 there is a bijection between $H(S)$ and $H\left(S_{* \ell}\right)$ which may be described as follows. Let $X=$ $s_{d}^{-1}(S)$. Consider $z=(a, b, i, j) \in H(S)$. Here $i, j \in[d]$. Write $a=r \ell+s, b=$ $r^{\prime} \ell+s^{\prime}, s, s^{\prime} \in[\ell]$. Then $\mathfrak{h}_{X, d}^{-1} \operatorname{maps}(a, b, i, j)$ to $(a d+i, b d+j)=(r(d \ell)+$ $\left.s d+i, r^{\prime}(d \ell)+s^{\prime} d+j\right) \in H(X)$ which by $\mathfrak{h}_{X, d \ell}$ is mapped to $z^{\prime}=\left(r, r^{\prime}, s d+\right.$ $\left.i, s^{\prime} d+j\right)$. This bijection restricts to a bijection between the $(\ell, 0)$-hooks in $H(S)$ and the $(1,0)$-hooks in $H\left(S_{* \ell}\right)$. More generally it restricts to a bijection between the $(k \ell, 0)$-hooks in $H(S)$ and the $(k, 0)$-hooks in $H\left(S_{* \ell}\right)$. This may also be applied to the $d$-symbol $C_{(\ell)}(S)$ having the property that $C_{(\ell)}(S)_{* \ell}=C\left(S_{* \ell}\right)$. (Thus $C_{(\ell)}(S)=s_{d}\left(s_{d \ell}^{-1}\left(C\left(S_{* \ell}\right)\right)\right)$.) It shows that $C_{(\ell)}(S)$ has no $(\ell, 0)$-hooks and it is thus called the $\ell$-core of $S$. It is really the $(\ell, 0)$-core of $S$. The reader should notice that the quotient $Q_{\ell}(S)$ is a $d \ell$-symbol, whereas the core $C_{(\ell)}(S)$ is a $d$-symbol. (Subscripts with brackets are used for $d$-symbols and subscripts without brackets for $d \ell$-symbols.)

Here is an example:

Example 5.1 Choose $d=2, \ell=3$. Consider $S=\left(X_{0}, X_{1}\right)$ with $X_{0}=\{9,7,4,2\}$, $X_{1}=\{3,1,0\}$. We get $X=s_{2}^{-1}(S)=\{18,14,8,7,4,3,1\}$ and $p(S)=p(X)=$ $(12,9,4,4,2,2,1)$. We compute $S_{* 3}=\left(X_{0}^{\prime}, X_{1}^{\prime}, \ldots, X_{5}^{\prime}\right)$, where

$$
\begin{array}{ll}
X_{0}^{\prime}=\{3\}, \quad X_{1}^{\prime}=\{1,0\}, \quad X_{2}^{\prime}=\{2,1\}, \quad X_{3}^{\prime}=\{0\}, \\
X_{4}^{\prime}=\{0\}, \quad X_{5}^{\prime}=\emptyset
\end{array}
$$

and get as balanced 3-quotient of $S$ the 6-symbol

$$
Q_{3}(S)=t_{6}((3),(0),(1,1),(0),(0),(0))=\left(Y_{0}, Y_{1}, \ldots, Y_{5}\right),
$$


where

$$
\begin{array}{ll}
Y_{0}=\{4,0\}, & Y_{1}=\{1,0\}, \quad Y_{2}=\{2,1\}, \quad Y_{3}=\{1,0\}, \\
Y_{4}=\{1,0\}, & Y_{5}=\{1,0\} .
\end{array}
$$

We have $C\left(S_{* 3}\right)=([1],[2],[2],[1],[1],[0])$, and $s_{6}^{-1}\left(C\left(S_{* 3}\right)\right)=\{8,7,4,3,2,1,0\}$ so that $C_{(3)}(S)$ is the 2-symbol

$$
C_{(3)}(S)=(\{4,2,1,0\},\{3,1,0\}) .
$$

Let $\delta=\left(c_{0}, c_{1}, \ldots, c_{d-1} ; m\right)$ be a $d$-hook data tuple. Consider the $d \ell$-hook data tuple $\delta_{* \ell}=\left(c_{1}^{\prime}, \ldots, c_{d \ell-1}^{\prime} ; m \ell\right)$ where we have $c_{i^{\prime}}^{\prime}=s m+c_{i}$ if $i^{\prime}=s d+i$. Thus

$$
\begin{aligned}
\delta_{* \ell}= & \left(c_{0}, c_{1}, \ldots, c_{d-1},\right. \\
& m+c_{0}, m+c_{1}, \ldots, m+c_{d-1}, \\
& 2 m+c_{0}, 2 m+c_{1}, \ldots, 2 m+c_{d-1}, \\
& \ldots, \\
& \left.(\ell-1) m+c_{0}, \ldots,(\ell-1) m+c_{d-1} ; m \ell\right) .
\end{aligned}
$$

We then find that if $z \in H(S)$ is mapped to $z^{\prime} \in H\left(S_{* \ell}\right)$ then $h^{\delta}(z)=h^{\delta_{* \ell}}\left(z^{\prime}\right)$. Indeed, if $z=(a, b, i, j)$ is mapped to $z^{\prime}=\left(r, r^{\prime}, s d+i, s^{\prime} d+j\right)$ in the above notation then

$$
\begin{aligned}
h^{\delta}(z) & =(a-b) m+c_{i}-c_{j} \\
& =\left(r-r^{\prime}\right) m \ell+\left(s m+c_{i}\right)-\left(s^{\prime} m+c_{j}\right)=h^{\delta_{* \ell}}\left(z^{\prime}\right) .
\end{aligned}
$$

Thus

$$
\mathcal{H}^{\delta}(S)=\mathcal{H}^{\delta_{* \ell}}\left(S_{* \ell}\right) .
$$

This may be applied to $\delta=\delta^{o}=(0,0, \ldots, 0 ; 1)$, the minimal $d$-hook data tuple (Remark 3.1). The length $h^{\delta^{o}}(z)$ of the hook $z=(a, b, i, j) \in H(S)$ is then $h(z)=$ $a-b$. Thus $\mathcal{H}(S):=\mathcal{H}^{\delta^{o}}(S)$ is just the multiset of hook lengths of $S$, as defined in [2], including short hooks of length 0 .

We have

$$
\delta_{* \ell}^{o}=(0, \ldots, 0,1, \ldots, 1, \ldots, \ell-1, \ldots, \ell-1 ; \ell) .
$$

Now apply Theorem 3.3 to the $d \ell$-symbol $S_{* \ell}$ and $\delta_{* \ell}^{o}$. The balanced quotient $Q$ in the theorem is just the balanced $\ell$-quotient of $S_{* \ell}$, i.e., $Q=Q_{\ell}(S)$. The core in the theorem is $C\left(S_{* \ell}\right)$. By definition of $C_{(\ell)}(S)$ we have $C_{(\ell)}(S)_{* \ell}=C\left(S_{* \ell}\right)$. Corresponding to $\delta_{S}$ in the theorem, we have here

$$
\begin{aligned}
\delta_{\ell, S}= & x_{0,0} \ell, \ldots, x_{d-1,0} \ell, \\
& 1+x_{0,1} \ell, \ldots, 1+x_{d-1,1} \ell, \\
& \ldots, \\
& \left.(\ell-1)+x_{0, \ell-1} \ell, \ldots,(\ell-1)+x_{d-1, \ell-1} \ell ; \ell\right),
\end{aligned}
$$


where $x_{i, j}$ is the number of elements in $X_{i}$ which are congruent to $j$ modulo $\ell$. By the above $\mathcal{H}^{\delta^{o}}(S)=\mathcal{H}^{\delta_{* \ell}^{o}}\left(S_{* \ell}\right)$ and $\mathcal{H}^{\delta^{o}}\left(C_{(\ell)}(S)\right)=\mathcal{H}^{\delta_{* \ell}^{o}}\left(C\left(S_{* \ell}\right)\right)$. As in Theorem 4.3 we see that the hook lengths are nonnegative. Thus we obtain

Theorem 5.2 Let $S=\left(X_{0}, X_{1}, \ldots, X_{d-1}\right)$ be a d-symbol. Let $C=C_{(\ell)}(S)$ be the $\ell$-core of $S$ and let $Q=Q_{\ell}(S)$ be the balanced $\ell$-quotient of $S$. Then

$$
\mathcal{H}(S)=\operatorname{abs}\left(\mathcal{H}^{\delta_{\ell, S}}(Q)\right) \cup \mathcal{H}(C)
$$

where $\operatorname{abs}\left(\mathcal{H}^{\delta_{\ell, S}}(Q)\right)$ is the multiset of all $\left|h^{\delta_{\ell, S}}(z)\right|, z \in H(Q)$.

We continue Example 5.1.

Example 5.3 We have $d=2, \ell=3, S=\left(X_{0}, X_{1}\right)$ with $X_{0}=\{9,7,4,2\}, X_{1}=$ $\{3,1,0\}$ and $p(S)=(12,9,4,4,2,2,1)$. The hook lengths (including 3 short hooks of length 0$)$ of $S$ may conveniently be read off the 2-abacus for $X=s_{2}^{-1}(S)$ and recorded systematically in the Young diagram of $p(S)$. The 2-abacus representation of $X$ (where the subscripts indicate the elements of $X_{0}$ and $X_{1}$ ) is

$\begin{array}{cc}0 & \mathbf{1}_{0} \\ 2 & \mathbf{3}_{1} \\ \mathbf{4}_{2} & 5 \\ 6 & \mathbf{7}_{3} \\ \mathbf{8}_{4} & 9 \\ 10 & 11 \\ 12 & 13 \\ \mathbf{1 4}_{7} & 15 \\ 16 & 17 \\ \mathbf{1 8}_{9} & 19\end{array}$

The hook lengths of $S$, i.e., the elements of $\mathcal{H}(S)$ :

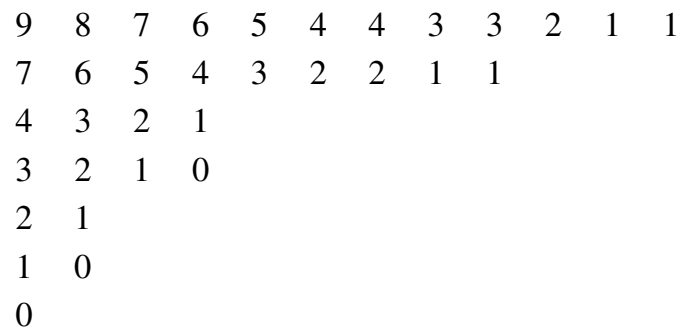

We calculated $C=C_{(3)}(S)=(\{4,2,1,0\},\{3,1,0\})$ and get $p(C)=(2,2)$ with hook lengths 
so that $\mathcal{H}(C)=\{2,1,1,0\}$. We also calculated $Q=Q_{3}(S)=\left(Y_{0}, Y_{1}, \ldots, Y_{5}\right)$ where $Y_{0}=\{4,0\}, Y_{1}=\{1,0\}, Y_{2}=\{2,1\}, Y_{3}=\{1,0\}, Y_{4}=\{1,0\}, Y_{5}=\{1,0\}$. We put $Q$ on the 6-abacus

$\begin{array}{cccccc}\mathbf{0}_{0} & \mathbf{1}_{0} & 2 & \mathbf{3}_{0} & \mathbf{4}_{0} & \mathbf{5}_{0} \\ 6 & \mathbf{7}_{1} & \mathbf{8}_{1} & \mathbf{9}_{1} & \mathbf{1 0}_{1} & \mathbf{1 1}_{1} \\ 12 & 13 & \mathbf{1 4}_{2} & 15 & 16 & 17 \\ 18 & 19 & 20 & 21 & 22 & 23 \\ \mathbf{2 4}_{4} & 25 & \ldots & & & \end{array}$

The hook data tuple $\delta_{3, S}$ in Theorem 5.2 is here $\delta_{3, S}=(3,6,7,4,5,2 ; 3)$. We record the $\delta_{3, S}$-hook lengths of $Q$ in the Young diagram of $p(Q)=(13,4,2,2,2,2,2,1,1,1)$

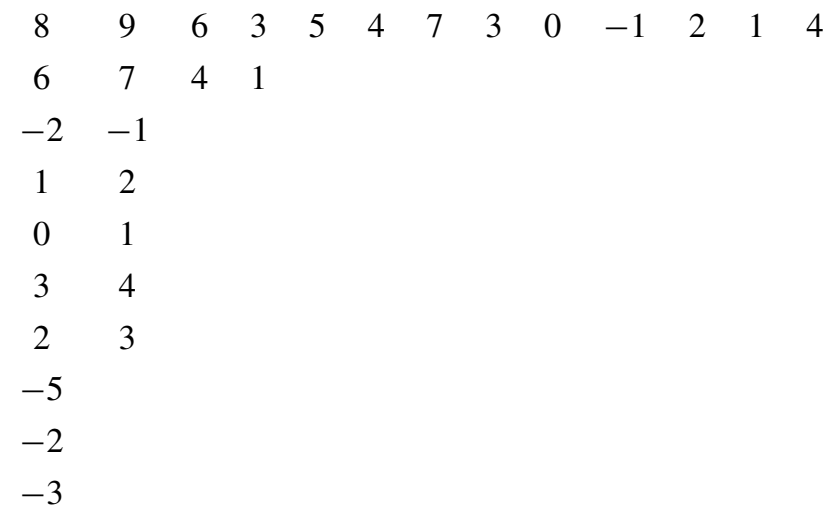

The set of absolute values of these elements form $\operatorname{abs}\left(\mathcal{H}^{\delta_{3}, S}(Q)\right)$ and we see that $\mathcal{H}(S)=\operatorname{abs}\left(\mathcal{H}^{\delta_{3, s}}(Q)\right) \cup \mathcal{H}(C)$.

We want to generalize this to $(\ell, e)$-cores and $(\ell, e)$-quotients, $e \in[d]$, the above Theorem 5.2 being the case $e=0$. Involving a suitable permutation of $\mathbb{N}_{0}$, this is done easily. This permutation $\sigma=\sigma_{d, \ell, e}$ is defined as follows. Any $n \in \mathbb{N}_{0}$ may be written uniquely as $n=r(d \ell)+s d+t, \quad r \in \mathbb{N}_{0}, s \in[\ell], t \in[d]$. Then $\sigma_{d, \ell, e}(n)=$ $r(d \ell)+s d+(t+r e)_{[d]}$. The $(\ell, e)$-twist $\sigma(S)$ of the $d$-symbol $S$ is defined as $\sigma(S)=$ $s_{d} \sigma\left(s_{d}^{-1}(S)\right)$. Note that if $S=\left(X_{0}, X_{1}, \ldots, X_{d-1}\right)$, then the $d$-symbol $\sigma(S)$ is not equal to $\left(\sigma\left(X_{0}\right), \sigma\left(X_{1}\right), \ldots, \sigma\left(X_{d-1}\right)\right)$. But if $X=s_{d}^{-1}(S)$ is the $\beta$-set of $S$, then $\sigma(X)$ is the $\beta$-set of $\sigma(S)$. It should be noted that similar cores and quotients occur in [2, Proof of 3.4].

Let us consider a long hook $z=(a, b, i, j) \in H(S)$, i.e., we have $a>b$. Write $a=r \ell+s, b=r^{\prime} \ell+s^{\prime}, s, s^{\prime} \in[\ell]$. Then 


$$
\begin{aligned}
& (a, b, i, j) \in H(S) \\
& \mathfrak{h}_{X, d}^{-1} \\
& (a d+i, b d+j)=\left(r(d \ell)+s d+i, r^{\prime}(d \ell)+s^{\prime} d+j\right) \in H(X) \\
& \downarrow \sigma \\
& (\sigma(a d+i), \sigma(b d+j))= \\
& \left(r(d \ell)+s d+(i+r e)_{[d]}, r^{\prime}(d \ell)+s^{\prime} d+\left(j+r^{\prime} e\right)_{[d]}\right) \in H(\sigma(X)) \\
& \downarrow \mathfrak{h}_{X, d} \\
& z^{\prime}=\left(a, b,(i+r e)_{[d]},\left(j+r^{\prime} e\right)_{[d]}\right) \in H(\sigma(S))
\end{aligned}
$$

Here it should be noted that since $z$ is long, then $\sigma(a d+i)>\sigma(b d+j)$ so that $(\sigma(a d+i), \sigma(b d+j)) \in H(\sigma(X))$. Therefore the above is a bijection between the sets $H_{>0}(S)$ and $H_{>0}(\sigma(S))$ of long hooks. There is in general no bijection for short hooks; their number may differ!

It follows from the definitions that if $\delta^{o}=(0,0, \ldots, 0 ; 1)$ is the minimal $d$-hook data tuple, then corresponding long hooks in $S$ and $\sigma(S)$ have the same $\delta^{o}$-length. Now $\delta^{o}$-lengths are always nonnegative and the long hooks are exactly those of nonzero $\delta^{o}$-length. Thus

$$
\mathcal{H}_{>0}^{\delta^{o}}(S)=\mathcal{H}_{>0}^{\delta^{o}}(\sigma(S))
$$

If $z=(a, b, i, j) \in H(S)$ is an $(\ell, e)$-hook, i.e., $a-b=\ell,(j-i)_{[d]}=e$, then in the above notation $r^{\prime}=r-1, s=s^{\prime}$ and $(a, b, i, j)$ is mapped to $z^{\prime}=(a, b,(i+$ $\left.r e)_{[d]},(j+(r-1) e)_{[d]}\right) \in H(\sigma(S)$. Since

$$
(j+(r-1) e)_{[d]}-\left((i+r e)_{[d]}\right)_{[d]}=(j-i-e)_{[d]}=0_{[d]}
$$

we see that $z^{\prime}$ is an $(\ell, 0)$-hook in $H(\sigma(S))$.

This shows that the $d$-symbol $C_{(\ell, e)}(S)$ satisfying that $\sigma\left(C_{(\ell, e)}(S)\right)=$ $C_{(\ell)}(\sigma(S))$ is obtained from $S$ by removing all $(\ell, e)$-hooks. (Thus $C_{(\ell, e)}(S)=$ $s_{d} \sigma^{-1} s_{d}^{-1}\left(C_{(\ell)}(\sigma(S))\right)=s_{d} \sigma^{-1} s_{d \ell}^{-1}\left(C\left(\sigma(S)_{* \ell}\right)\right)$.) We call $C=C_{(\ell, e)}(S)$ the $(\ell, e)$ core of $S$. We also need to define a (balanced) $(\ell, e)$-quotient $Q=Q_{\ell, e}(S)$ of $S$. To do this we consider the $d \ell$-symbol $S_{* \ell, e}$ defined by

$$
S_{* \ell, e}:=s_{d \ell} \sigma\left(s_{d}^{-1}(S)\right) .
$$

Thus $S_{* \ell, e}$ is the $d \ell$-symbol associated to the $\beta$-set $\sigma(X)$. If again $\sigma(S)=$ $s_{d} \sigma\left(s_{d}^{-1}(S)\right)$ is the $(\ell, e)$-twist of $S$, then by definition

$$
\sigma(S)_{* \ell}=S_{* \ell, e} .
$$

We define $Q=Q_{\ell, e}(S):=Q\left(S_{* \ell, e}\right)=Q\left(\sigma(S)_{* \ell}\right)$, which is a $d \ell$-symbol. 
We now apply Theorem 5.2 to the $d$-symbol $\sigma(S)$. We get for a suitable $d \ell$-hook data tuple $\delta=\delta_{\ell, \sigma(S)}$, defined in analogy with $\delta_{\ell, S}$ above,

$$
\mathcal{H}(\sigma(S))=\operatorname{abs}\left(\mathcal{H}^{\delta}(Q)\right) \cup \mathcal{H}(C),
$$

where $\operatorname{abs}\left(\mathcal{H}^{\delta}(Q)\right)$ is the multiset of all $\left|h^{\delta}(z)\right|, z \in H(Q)$. In this multiset equality we remove all occurrences of 0 and get

$$
\mathcal{H}_{>0}(\sigma(S))=\operatorname{abs}\left(\mathcal{H}_{>0}^{\delta}(Q)\right) \cup \mathcal{H}_{>0}\left(\sigma(S)_{(\ell)}\right) .
$$

By the above $\mathcal{H}_{>0}(\sigma(S))=\mathcal{H}_{>0}(S)$ and $\mathcal{H}_{>0}\left(\sigma(S)_{(\ell)}\right)=\mathcal{H}_{>0}\left(S_{(\ell, e)}\right)$. We have shown

Theorem 5.4 Suppose that $S=\left(X_{0}, X_{1}, \ldots, X_{d-1}\right)$ is a $d$-symbol. Let $C=$ $C_{(\ell, e)}(S)$ be the $(\ell, e)$-core of $S, Q=Q_{\ell, e}(S)$ the balanced $(\ell, e)$-quotient of $S$ and $\delta=\delta_{\ell, \sigma(S)}$. Then

$$
\mathcal{H}_{>0}(S)=\operatorname{abs}\left(\mathcal{H}_{>0}^{\delta}(Q)\right) \cup \mathcal{H}_{>0}(C),
$$

where $\operatorname{abs}\left(\mathcal{H}_{>0}^{\delta}(Q)\right)$ is the multiset of all non-zero $\left|h^{\delta}(z)\right|, z \in H(Q)$.

Remark 5.5 In analogy with Corollary 4.12 we may use the Theorems 5.2 and 5.4 to prove relative hook formulas for unipotent degrees. See [4, Proposition (8.9)] for unipotent character degrees in finite classical groups or more generally [2, 3.12].

Example 5.6 Let us consider the case $d=2, \ell=3, e=1$. The permutation $\sigma=$ $\sigma_{2,3,1}$ is a product of transpositions:

$$
\sigma=\prod_{k \text { odd }}(6 k, 6 k+1)(6 k+2,6 k+3)(6 k+4,6 k+5) .
$$

We want to apply Theorem 5.4 to the 2-symbol $S=\left(X_{0}, X_{1}\right)$ with $X_{0}=\{9,7,4,2\}$, $X_{1}=\{3,1,0\}$. We have $X=s_{2}^{-1}(S)=\{18,14,8,7,4,3,1\}$. Thus $\sigma(X)=\{19,14$, $9,6,4,3,1\}$ and

$$
\sigma(S)=s_{2}(\sigma(X))=(\{7,3,2\},\{9,4,1,0\})
$$

Putting $\sigma(S)$ on the $d \ell=6$-abacus we get in analogy with Example 5.1 that

$$
\sigma(S)_{* 3}=S_{* 3,1}=(\{1\},\{3,0\},\{2\},\{1,0\},\{0\}, \emptyset)
$$

and the balanced quotient is

$$
Q=Q_{3,1}(S)=(\{1\},\{2\},\{2\},\{0\},\{0\},\{0\}) .
$$

We have $C\left(\sigma(S)_{* 3}\right)=([1],[2],[1],[2][1], \emptyset)$. Thus $\sigma(S)$ has 3-core

$$
C=C_{(3,1)}(S)=s_{2} \sigma^{-1} s_{6}^{-1}\left(C\left(\sigma(S)_{* 3}\right)\right)=([5],[2])
$$


with

$$
\mathcal{H}_{>0}(C)=\{2,1,1\} .
$$

We have $\delta_{\ell, \sigma(S)}=(3,6,4,7,5,2 ; 3)$ and list the $\delta_{\ell, \sigma(S)}$-hooks of $Q$ in the Young diagram of $p(Q)=(9,9,3,3,3,3)$ :

$\begin{array}{ccccccccc}7 & 4 & 6 & 1 & 3 & 0 & 2 & 5 & 1 \\ 9 & 6 & 8 & 3 & 5 & 2 & 4 & 7 & 3 \\ 3 & 0 & 2 & & & & & & \\ -1 & -4 & -2 & & & & & & \\ 2 & -1 & 1 & & & & & & \\ 4 & 1 & 3 & & & & & & \end{array}$

The union of the multiset of the non-zero absolute values of these hook lengths and $\mathcal{H}_{>0}(C)$ coincides with the multiset of hook lengths of $S$ listed in the Young diagram of $p(S)$ in Example 5.3. This is in accordance with Theorem 5.4.

Acknowledgement The authors wish to thank Gabriel Navarro for a discussion leading to this investigation.

\section{References}

1. James, G., Kerber, A.: The Representation Theory of the Symmetric Group. Encyclopedia of Mathematics and its Applications, vol. 16. Addison-Wesley, Reading (1981)

2. Malle, G.: Unipotente Grade imprimitiver komplexer Spiegelungsgruppen. J. Algebra 177, 768-826 (1995)

3. Malle, G., Navarro, G.: Blocks with equal height zero degrees. Trans. Am. Math. Soc. 363, 6647-6669 (2011)

4. Olsson, J.B.: Combinatorics and Representations of Finite Groups. Vorlesungen aus dem FB Mathematik der Univ. Essen, vol. 20 (1993) (This book is freely available at the author's homepage) 